\title{
Le tragi-comique, la migration urbaine et la couleur glocale dans Bleu Blanc Rouge et Black Bazar d'Alain Mabanckou
}

\section{The Tragi-comic, Urban Migration and Glocal Colour in Bleu Blanc Rouge and Black Bazar by Alain Mabanckou}

Petr Vurm [vurm@phil.muni.cz]

Masarykova univerzita, République tchèque

\section{RÉSUMÉ}

Notre article se pose pour but de démontrer quelques-unes des stratégies narratives et rhétoriques mises en place par le romancier, journaliste, poète et académicien contemporain, né en République du Congo, Alain Mabanckou. Plus précisément, nous essaierons de discuter la tension dynamique entre les tribulations tragiques et les aboutissements comiques des aventures des protagonistes principaux, ainsi que d'analyser des éléments de l'humour dans leur discours. Nous nous concentrerons principalement sur deux ouvrages, le premier roman de Mabanckou, Bleu Blanc Rouge (1998) et un autre, assez récent, Black Bazar (2009). Notre hypothèse de travail est que la tension susmentionnée est influencée ou même découle d'une corrélation avec une hétérotopie foucaultienne, et avec des transitions dynamiques entre deux topoi principaux : celui de Paris représentant la Métropole et celui de Brazzaville, Pointe-Noire ou un village typique ordinaire quelque part dans la République du Congo, symbolisant le chronotope postcolonial, chargé de son passé colonial.

\section{MotS-CLÉS}

Mabanckou ; humour ; tragi-comique ; migration ; ville ; glocalisation ; Black Bazar ; Bleu Blanc Rouge

\begin{abstract}
Our paper tries to show some of the narrative and rhetoric strategies used by the novelist, journalist, poet and important scholar born in the Republic of Congo, Alain Mabanckou. More exactly, we shall try to discuss the dynamic tension between the tragic tribulations and comical outcomes of the adventures of the main characters, as well as to analyze some of the elements of humour in their discours. We shall concentrate on two works principally, the first novel by Mabanckou, Bleu Blanc Rouge (1998) and another quite recent one, Black Bazar (2009). Our working hypothesis is that the above-mentioned tension is influenced and ensues from a correlation with a Foucauldian heterotopia, with dynamique transitions between two main topoi: that of Paris, representing the Metropole and that of Brazzaville, Pointe-Noire or a typical commonplace village somewhere in the Republic of Congo, symbolising the postcolonial chronotope, laden with its colonial past.
\end{abstract}

\section{KEYWORDS}

Mabanckou; humor; tragi-comic; migration; city; glocalisation; Black Bazar; Bleu Blanc Rouge 
Alain Mabanckou est un romancier, journaliste, poète et académicien contemporain, né en République du Congo. Il représente la nouvelle génération d'auteurs qui ont participé au renouveau des Lettres africaines que l’on peut constater à la fin des années 1990. Ceux-ci sont souvent associés de façon très libre au courant de l'Afrique sur Seine. Son premier roman, Bleu Blanc Rouge, qui obtient en 1998 le Grand Prix de l'Afrique noire, a à été l'un des plus remarqués. Ce que la critique apprécie chez cet auteur, c'est qu'il est à la fois essayiste, journaliste, poète et professeur de littérature, ainsi ses romans reprennent beaucoup de sujets de son écriture essayistique et journalistique, mais sous une autre lumière, comme seulement le roman le permet, un peu à la manière de Kundera, qui dit dans son Art du roman :

Découvrir ce que seul un roman peut découvrir, c'est la seule raison dêttre d'un roman. Le roman qui ne découvre pas une portion jusqu'alors inconnue de l'existence est immoral. La connaissance est la seule morale du roman. $(1986: 16)$

Lécriture mabanckouienne se trouve à la croisée des littératures congolaise, africaine, française, postcoloniale et « migrante » et donc se prête facilement à une critique littéraire disons contemporaine, chacun peut y trouver un sujet favori. On pourrait analyser facilement le rapport des identités noire et blanche la représentation de ce celles-ci au prolongement de la négritude ou du «peau noires, masques blancs » de Franz Fanon, ainsi qu’on pourrait s'arrêter sur la problématique de l'exil et du voyage, ou dans notre cas, avant et surtout du mythe urbain de la ville de Paris. Cela a déjà été fait, notamment dans Moudileno 2006, Simédoh 2012 ou Cazenave 2004. Notre propos sera d'aborder un sujet qui n’a pas été traité de manière systématique, à notre connaissance. Il s'agit des aspects du tragique et du comique, ou du tragi-comique et de toute une gamme de notions corollaires, tel que l'humour le rire et l'ironie chez cet auteur. Plus précisément, nous essaierons de discuter la tension dynamique entre les tribulations tragiques et les aboutissements comiques des aventures des protagonistes principaux, ainsi que d'analyser des éléments de l'humour dans leur discours. Nous nous concentrerons principalement sur deux ouvrages, le premier roman de Mabanckou, Bleu Blanc Rouge (1998) et un autre assez récent, Black Bazar (2009). Notre hypothèse de travail est que la tension susmentionnée est influencée ou même découle d'une corrélation avec l'hétérotopie foucaultienne ${ }^{1}$ mutuellement exclusive, et avec des transitions entre deux topoi principaux : celui de Paris représentant la Métropole et celui de Brazzaville, Pointe-Noire ou un village typique ordinaire quelque part dans la République du Congo, symbolisant le chronotope postcolonial, chargé de son passé colonial. Nous croyons pouvoir démontrer que l'expérience migrante de l'auteur et son vécu au sens le plus profond du terme, telle qu'il la distille finalement dans ses romans à travers ses personnages et leurs discours, est indispensable à l'expression du comique et au rire du lecteur. L'un des nos propos principaux sera également de démontrer une certaine continuité et développement

1 Les hétérotopies inquiètent, sans doute parce qu'elles empêchent de nommer ceci et cela, parce qu’elles ruinent d'avance la "syntaxe ", et pas seulement celle qui construit les phrases, - celle moins manifeste qui fait " tenir ensemble » (à côté et en face les unes des autres) les mots et les choses. C'est pourquoi les utopies permettent les fables et les discours : elles sont dans le droit fil du langage, dans la dimension fondamentale de la fabula, les hétérotopies [...] dessèchent le propos, arrêtent les mots sur eux-mêmes ; contestent, dès sa racine, toute possibilité de grammaire ; elles dénouent les mythes et frappent de stérilité le lyrisme des phrases. (Foucault $1966: 9-10$ ). 
dans l'écriture de l'Afrique subsaharienne, comme ni la migration Afrique-Europe ni l'humour ne sont pas nouveau dans celle-ci.

Premièrement, abordons la migration et le voyage. La littérature africaine francophone ne manque pas de récits autour du thème du voyage à la métropole européenne. De Kocoumbo létudiant noir d’Âké Loba, en passant par les Mirages de Paris du Sénégalais Ousmane Socé, La Trahison de Marianne de Bernard Nganga, Un Nègre à Paris de Bernard Dadié, L'Aventure ambiguë de Cheik Hamidou Kane, Le baobab fou de Ken Bugul ou La Nouvelle romance d'Henri Lopes. L'apparition, dans les années 1960 et 1970, des diverses illustrations romanesques de ce voyages, en a rapidement fait un thème privilégié de la littérature africaine. Mais la France avait relativement disparu de la fiction africaine après les indépendances, au profit d'un recentrement su des thèmes inspirés des réalités sociopolitiques de nouvelles nations africaines. Or, depuis une dizaine d'années, une nouvelle génération d'écrivains redonne une place centrale à la France dans la fiction africaine et soumettant le topos du voyage à la métropole à dess retournements inédits. Inaugurée par Le Petit prince de Belleville de Calixthe Beyala en 1990, cette tendance s'est accélérée tout au long de la décennie. Entre 1995 et 2000, on relève ainsi plus d’une dizaine de récits ayant pour sujet la migration et le voyage. Rien que dans les dix années de la fin du siècle une dizaine de titres publiés renvoient au même sujet : Le Paradis du Nord de Jean Roger Essomba (2000), Ici s'arrête le voyage de Léandre-Alain Baker (1996), Assèze l'Africaine de Calixthe Beyala (1994), Dans la peau d'un sans-papier d'Aboubacar Diop (1997), L'Impasse de Daniel Biyaoula (1996) et Bleu Blanc Rouge d'Alain Mabanckou (1998) (Moudileno 2006 : 107).

Certains critques, ocomme Jacques Chevrier, constatent une banalisation du thème de l'émigration en France dans la fiction récente. D’autres, comme Benetta Jules-Rosette, y voient la continuité d'un parisianisme lancé dès le milieu du siècle par les intellectuels de Présence africaine. D'autres n'hésitent pas à parler de l'émergence d'un roman de l'émigration, comparable pour l'Afrique subsaharienne à l'avènement de la littérature beure dans les années 1980 (Moudileno 2006 : 108). Si le thème du voyage à Paris est loin d'être nouveau, il s'agit de voir comment Mabanckou le renouvelle et l'adapte au contexte des années 1990 et 2000 mais aussi comment il l'emploie dans ses stratégies du tragique et du comique.

Deuxièmement, c'est le comique, l'humour et l'ironie dans la littérature africaine subsaharienne, où il faut chercher une certaine tradition dont Mabanckou serait le continuateur. En comparaison avec la thématique du voyage, l'humour, tel que nous l'imaginons, n'est pas si fréquent dans les littératures d'Afrique subsaharienne, quoique il ne soit pas tout à fait absent non plus. A la différence de l'Europe, le comique est rarement frivole et non engagé, lécrivain fait rarement de l'humour pour l'humour, l'effect de comique fonctionne souvent assez différemment au fond des situations dramatiques, tristes, voire tragiques qu'est le contexte de l'Afrique. Au moins deux grands auteurs désormais classiques s'offrent dans ce sens. Mongo Beti et Ferdinand Oyono, les deux étant camerounais.

W. A. Pannenborg nous semble avoir donné une des meilleures définitions d'humour convenable à notre propos :

Le terme de l'humour, signifie raillerie bienveillante. Le sentiment du risible sur fond de sympathie. La disposition à la raillerie indulgente[...] est essentielle à l'humour, autrement dit, il faut que la raillerie frôle la compassion, sinon il n'est pas question d'humour. Par contre la satire est 
une raillerie outrageante, haineuse, dure, sans charité, on pourrait dire, suivant une variante de la formule précédente. Le sentiment du risible sur fond d'antipathie, ce qu'il y a dans l'humour d'indulgent, de tolérant, manque dans la satire. Il en résulte que l'humour comporte on ne sait quoi de relatif, la satire au contraire quelque chose d'absolu, la condamnation sans réserves qui ne tient pas compte des circonstances atténuantes. (Pannenborg $2005: 129$ )

Chez Oyono, l'humour cherche à exploiter dans l'objet raillé son aspect insolite, inhabituel, et qui prête au rire ; il est tantôt bienveillant et tolérant, tantôt destructeur, selon l'image que le romancier veut créer de son adversaire ; il n'épargne personne : ni l'administrateur colonial et son entourage, ni le missionnaire, ni même le colonisé dont l'auteur semble prendre la défense.

Le vieux nègre et la médaille, publié en 1956, se concentre sur la date symbolique du 14 juillet, fêtée dans un district éloigné. Ce jour-là, Meka, qui a donné du terrain aux missionnaires pour leur église et dont les deux fils sont morts à la guerre, est d'abord heureux d'être honoré par une médaille de reconnaissance de la France, à laquelle tous ses proches applaudissent. En deux jours, après une cérémonie qui devient une parodie, le vieil homme prend conscience que ce 14 juillet n'est en fait qu'une mise en scène hypocrite des pouvoirs coloniaux qui parlent d'amitié en maintenant une stricte exclusion des colonisés. La solidarité africaine qui l'entoure à la fin du roman constitue un contrepoint politique et, avec la fierté retrouvée du peuple colonisé, une réponse à la colonisation des Blancs.

Le rire, à travers toutes les différentes formes qu'il peut prendre, à savoir la dérision, la parodie, le sarcasme, le grotesque, par le biais de l'humour et de l'ironie qui sont les manifestations apparentes, est un outil à la fois de la contestation, de la dédramatisation des situations tragiques, mais aussi une esthétique d'écriture en soi dans la représentation du réel, du sérieux, que ce soit au niveau social, politique ou de l'écriture elle-même qui joue à la fois sur la dissimulation, sur le carnavalesque, la polyphonie et le grotesque, pour finir à une poétique du rire.

Le rire est étroitement lié à une notion plus générale, celle du comique. La définition du comique est cependant une entreprise ambitieuse et peut-être impossible à mener à un terme pleinement satisfaisant ; elle touche aussi bien à l'esthétique qu'à la sociologie, l'histoire, la psychologie, voire la psychanalyse, l'anthropologie et la philosophie. Il nous semble plus prudent de nous en tenir à une approche esthétique, qui soit en même temps la plus inclusive possible : la notion de comique recouvre l'ensemble des procédés qui visent à susciter le rire.

A propos de l'humour, soulignons qu'en amont, c'est-à-dire dans les conditions de sa production, et en aval, à savoir sa réception, ses implications philosophiques, l'humour n'est peut-être pas toujours intrinsèquement lié au comique. D’une part, on peut arguer que l'humour dans ses manifestations artistiques naît d'une psychologie mélancolique voire dépressive ; d'autre part, on peut faire remarquer que l'humour mène peut-être, au-delà du rire, vers une philosophie et une éthique pessimistes, ou du moins résignées, qui prennent acte des contradictions inhérentes au monde et à toute activité humaine.

Ayant relevé quelques écueils définitionnels et théoriques liés au comique et à l'humour, passons enfin à la lecture de quelques exemples dans les oeuvres d'Alain Mabanckou. Chez l'auteur congolais en question, nous proposons de partir plutôt de la division classique du comique : comique de situations, de caractères, de mots, et de gestes, dont nous allons discuter les trois premiers et laisser le comique de gestes au théâtre. 
Renouant avec ce que nous venons de dire sur le versant mélancolique voire dépressif de l'humour, rappelons un fait peut-être paradoxal que le comique de Mabanckou naît, lui aussi, sur un fond bien tragique. Il s'agit à la fois de l'histoire collective(ment) tragique de l'Afrique cette histoire avec une grande hache de Perec - à commencer par la colonisation désastreuse du grand pays voisin République démocratique du Congo belge par la Belgique et plus particulièrement par Léopold II, qui a eu ses effets sur le Congo français, au cours de toute la colonisation. Rappelons à ce propos un petit détail consistant dans le fait que de nombreuses compagnies concessionnaires du Congo français étaient aux mains de nombreux actionnaires, dont Léopold II de Belgique, qui avait acheté des actions sous un faux nom. Ce fait, découvert après la mort du souverain belge, a choqué beaucoup les autorités françaises de l'époque, qui ont dû constater que leur colonie était exploitée par un pays étranger. A l'époque post-indépendantiste, les intérêts économiques des compagnies françaises aidaient à déclencher de nombreuses guerres civiles. Rappelons également les tragédie récentes, y compris le génocide au Rwanda et la guerre civile au Congo, motivée parmi d'autres par la ruée aux ressources tels que le cuivre, le cobalt, ou le coltan.

De l'autre côté, c'est l'histoire tragique individuelle, personnelle, souvent inséparable de la tragédie collective. La pauvreté et les guerres provoquent beaucoup de Congolais à une émigration vers l'Europe ou l'Amérique où ils ont souvent un statut inférieur au niveau social, du logement, du travail, du statut civil en général, et donc souvent aussi, ils sont amenés au crime. C’est exactement cet aspect socio-culturel des destinées de beaucoup de ses compatriotes qui inspire l'histoire de Bleu Blanc Rouge et de quelques autres romans à intrigue policière de Mabanckou, qui donc, malgré une certaine tonalité tragique apportent de nombreux éléments d'humour et ainsi deviennent tragi-comiques.

Bleu Blanc Rouge raconte l'histoire d'un jeune Congolais nommé Massala-Massala qui, séduit par l'exemple de ses compatriotes, quitte son pays natal pour la France, dans l'espoir d'en revenir, comme eux, transfiguré. Après maintes péripéties et désillusions, il se retrouve en prison en France, sur le point d’être expulsé par les autorités françaises. Ce roman est de facture assez simple : la narration du « voyage en France " s'y déploie en deux grandes sections, "Le pays » et « Paris », autour d'un prologue et d’un épilogue qui cadrent le réci rétrospectif des mésaventures du narrateur.

C’est précisément dans cette structure binaire que réside l'originalité du roman. Plutôt que de se concenter, comme beaucoup de ses prédécesseurs, sur l'expérience vécue en France, Mabanckou choisit d'accorder une part égale au pays d'origine et au pays dit d'accueil : conditions de départ et de retour apparaissent dès lors aussi importantes, sion plus, que le voyage lui-même. Comme le rappelle le sociologue Abdelmalek Sayad :

Toute étude des phénomènes de migration qui néglige les conditions d’origine des émigrés se condamne à ne donner du phénomène migratoire qu'une vue à la fois partielle et ethnocentrique : d'une part, comme si son existence commençait au momment où il arrive en France, c'est l'immigrant - et lui seul - et non l'émigré qui est pris en considération, et d'autre part, la problématique, explicite et implicite, est toujours celle de l'adaptation à la société d'accueil. $(1977: 59)$ 
Bleu Blanc Rouge est donc un des lieux exemplaires de la littérature africaine en général, et congolaise en particulier, où l'auteur propose et examine des fictions identitaires produites non plus uniquement dans les limites spécifiques d'une nation, comme chez Oyono, Bemba ou Labou Tansi, ni en se détournant radicalement de la métroplole coloniale, comme chez Lopes, mais dans une continuité de passages entre l'Afrique et l'Europe.

Observons maintenant de plus près les stratégies comiques employée dans son écriture. Abordons le comique de caractère d'abord : Mabanckou offre toute une gamme de personnages, dont quelques-uns sont forcément comiques. Il y a toute une typologie des personnages du point de vue de l'expérience migrante. La typologie serait difficile à établir, vu la diversité des caractères. Pourtant, il y a quelques types de base liés à l'expérience de la migration que nous pourrions énumérer :

- Pesonnages venant de la France / Personnages venant du Congo ou vivant au Congo / en France

- ceux qui n'ont jamais mis leur pied en dehors de leur village/pays

- ceux qui ont voyagé une seule fois

- ceux qui ont voyagé plusieurs fois

- ceux qui connaissent d'autres pays que le Congo et la France

- ceux qui comprennent les enjeux du monde global

- ceux qui ont une certaine ouverture d'esprit, qui, malgré le fait qu'ils n’ont jamais voyagé, leur permet de compenser ce défaut

Les personnages répertoriés dans cette typologie entrent en interaction dans le récit, ce qui a pour conséquence des chocs culturels et intellectuels et donc des situations comiques. Pour ne donner qu'un seul exemple, celui où « Moki le Parisien », personnage-sapeur, dandy congolais, entre en interaction avec les simples Congolais du type « villageois », de Pointe-Noire :

Par la suite, l’emploi du temps de Moki était fort simple. S’asseoir sous le manguier. Sortir lorsqu'il était l'invité des filles du quartier. Le matin, il lisait des journaux parisiens qu'il avait ramenés du nord : Ici Paris, Paris Match, Le Parisien... Il gardait sur lui son peignoir en soie avec des motifs en taffetas. Des jeunes gens du quartier, ses amis d'enfance,venaient lui couper les cheveux. Ils monnayaient ces services contre quelques objects de Paris. Et pas n'importe quels objets ! Moki les gratifiait de petites cartes de métro parisien. Ils exultaient. Il ne comprenaient pourtant pas ces itinéraires emmêlés, ces lignes numérotées et si entrecroisées qu’on aurait dit la carte hydrographique de la Chine. Ils surprenaient le Parisien lui-même. En effet, certains autochtones décrivaient avec un talent inégalable les lignes du métro, station par station, à croire qu'ils avaient séjourné à Paris. D’autres s'attribuaient pour pseudonymes les noms de ces stations. Tel se surnommait Saint-Placide. Tel autre Strasbourg-Saint-Denis. Tel autre encore Colonel Fabien ou Maubert-Mutualité. Ils adjoignaient à ces pseudonymes le mot « Monsieur ». Monsieur SaintPlacide, Monsieur Strasbourg-Saint-Denis, Monsieur Colonel Fabien, Monsieur Maubert-Mutualité. Moki leur fournissait également des cartes oranges vierges. Ils y collaient leur photographie et épataient les filles les plus naïves. (Mabanckou 1998 : 61) 
Le comique de situation consiste dans le choc de deux cultures disparates, dont l'une n'a jamais vu le métro, qui devient ici le symbole de la vraie Métropole. L'imitation du comportement du Parisien et du Grand Monde de la ville est comique parce qu'elle ressemble, grâce à sa fausse naïveté, au jeu des enfants.

Cet extrait, en même temps, introduit le comique de situation. Comme nous pouvons le voir, Mabanckou dépeint dans son récit des milieux incompatibles, voire hostiles, tels que les hauts lieux culturels de Paris, la banlieue de Paris et le monde rural du Congo. Les protagonistes, passant d'un milieu à l'autre, sont suceptibles de ne pas comprendre tous les codes qu'il faut respecter pour vivre et survivre dans un tel milieu, ce qui engendre presque automatiquement des situations comiques et le rire du lecteur. Dans ce type de comique de situation, il s'agit également d'un jeu complexe sur les connaissances et les savoir-vivre, le lecteur occidental ayant de la supériorité sur les personnages qui manquent la connaissance nécessaire. Ce faisant, l’auteur et le narrateur flattent le lecteur sur sa connaissance. Ainsi, il faut avoir le maximum de connaissances pour ne pas être dupe et pour, au contraire, duper. Ou du moins pour comprendre la duperie et pour rire avec son auteur.

Rappelons encore quelques éléments de ce jeu complexe : il y a Mabanckou-auteur, demiurge, qui insère dans ses récits les aspects de son autobiographie, voire d'autofiction - fiction d'un immigré qui n'aurait pas le talent d’écrivain et finirait, comme ses compatriotes dans un taudis de banlieu. Le narrateur, ensuite, serait proche de l'auteur, il serait hétérodiégétique, extradiégétique dans Bleu Blanc Rouge, homodiégétique et intradiégétique dans Black Bazar. Le lecteur occidental se trouve dans une position spéciale, il connait la plupart des codes du milieu occidental. Mais avant tout, à la différence des «villageois », natifs du Congo, il dispose d'un savoir général et surtout global, muni qu'il est d'un appareil critique et déductif, d'une expérience de lecture et d'interprétation, il est capable de détecter la raillerie et l'ironie, à la différence des protagonistes. Paradoxalement, et malgré les apparences, rappelons-le, il lui manque tout un volet de connaissances directes sur le Congo, sauf celles transmises par les médias, souvent fort déformées. En cela, il doit faire confiance au narrateur et il se trouve parfois dans une situation proche des personnages.

Finalement, passons au comique des mots et pour ce faire, parlons plus de Black Bazar. Au fur et à mesure que Mabanckou-écrivain mûrit, plus il sait maîtriser les mots et leur effet comique, comme c'est le cas dans son roman plus récent. Ici encore, c'est l'effet de surprise qui explique au bout du compte l'effet comique et le rire. Mabanckou amuse le lecteur par des mots insolites et leur rencontre inattendue, ce qui est en quelque sorte le germe de l'humour qui l'aide à développer ses idées.

Rappelons très brièvement l'histoire de Black Bazar. Le narrateur, surnommé par ses comparses Fessologue, nous livre sa vie à travers son écriture, de façon abrupte. C'est une vie ordinaire de sapeur congolais, ayant fui son pays natal, le Congo-Brazzaville, habitant Château-Rouge, quartier du 18e arrondissement de Paris, mais c'est l'histoire de la vie ordinaire faite de jalousies, de haines, de médiocrité, de douleurs. L’histoire se déroule pour la plupart du côté de Château-Rouge, entre émigrés. On partage le quotidien ordinaire du protagoniste, ses craintes et ses joies. On accompagne le bonhomme dans ses tribulations en ville, au marché Dejean, ses réflexions, son regard sur le monde, sur la négritude, on passe du temps avec lui, au Jip's, le café où il passe du temps à descendre des Pelforth, on écoute l'Arabe du coin louer son frère africain, 
le convaincre de mener le combat pour l'unité d'une Afrique forte, derrière le guide spirituel Mouammar Kadhafi.

Le narrateur, autrement dit le Fessologue, expert ès Faces B, c'est à dire de façon humoureuse, des derrière des femmes, est admirateur et amant de sa compatriote, Couleur d’origine. Malheureusement pour lui, Couleur d'origine le trompe avec un prétendu cousin, l'Hybride, jusqu'au jour où la belle Congolaise et son amoureux sont repartis en Afrique avec Henriette, la fille qu'elle aurait eu avec le Fessologue. Comme assez souvent, il y également le motif de l'écrivain fictif qui cadre la narration, à savoir que le narrateur, qui travaille assez épisodiquement dans une imprimerie, sest lancé dans lécriture de Black Bazar comme une sorte de chronique du quartier, ce faisant, il forme une pittoresque galerie de portraits à laquelle s'ajoutent Hippocrate le voisin de palier, l'Arabe du coin et ultérieurement Louis-Philippe Dalembert, un écrivain haitien chroniqué ici-même. La rencontre avec Louis-Philippe, écrivain haïtien, va lui donner l'envie décrire, il va commencer doucement à s'ouvrir au royaume des Lettres, il va s'acheter une machine à écrire et s'adonner, à la maison, dans les parcs, quand Couleur d'origine le pousse dehors, à sa nouvelle passion. Enfin, sa rencontre avec Sarah, peintre franco-belge, va lui permettre de s'ouvrir pleinement à toutes les littératures, en plus de celles d'Amérique latine que lui proposait Louis-Philippe et s'accomplir en tant qu'homme par le biais de son nouveau métier, écrivain.

Or, comme nous l'avons déjà constaté, il y a chez Mabanckou un riche panorama de personnages et de caractères dont la rencontre contribue à la constitution de l'effet de comique. En dehors de la blonde Sarah qui viendra initier le Fessologue à la littérature belge, tous les héros et anti-héros du roman sont des Africains de Paris, et le Fessologue est le plus élégant parmi eux, avec ses costumes Smalto, et ses chaussures Weston, bref un Sapeur.

Plus qu'ailleurs, la narration est fondée sur le comique de mots, le style décriture devient baroque et assez polyphonique et hybride, en amalgamant beaucoup de discours différents, celui des belles-lettres, des médias, de la science, des hommes noirs immigrés, des hommes blancs ou noirs racistes envers les immigrés. Mentionnons ici un exemple, le "monologue du Fessologue » qui est fondé sur le comique des mots, grâce à un discours qui parodie un discours scientifique :

Bon. Il se trouve que mon ex était née toute noiraude comme ça. Mon ex je l'avais surnommée Couleur d'origine. [...] Couleur d'origine avait un attribut im-pa-ra-ble. Son derrière. Son derrière bougeait dans le sens inverse des aiguilles d'une montre. Ce nétait pas n'importe quel derrière. Un derrière vitesse automatique. [...] J'avais trouvé chez Couleur d’origine le derrière de mes rêves. [...] Il y avait rien à dire sur la face $\mathrm{B}$ de Couleur d’origine. La plupart de mes potes m’appellent Fessologue. La manie de chasser du regard les chutes de reins des passantes et de me livrer par la suite à des analyses très poussées. [...] Mon pote Pierrot le Blanc du Petit Congo à chaque fois quand il a une nouvelle une copine, ne veut pas faire fausse route, tomber sur une capricieuse à cause d'une histoire du derrière mal fagoté. Il y a des derrières [...] où tu es carrément déçu. Est-ce que c'est vraiment un derrière que tu vois ? Parce qu'il n’a pas de gueule. Parce qu'il va à gauche qu'il ne va jamais à droite. Comme s'il y avait un danger de ce côté-là, comme il revient brusquement au point de départ et qu'il s'immobilise et qu'il s'aplatit sans élégance. [...] Il y a un autre type de derrière. Leur problème à eux est qu'ils remuent trop vite. Du haut 
en bas. [...] Il y a un autre type de derrière. Pire. Ils remuent par à-coups. Ils sont épileptiques. [...] (transcrit de la pièce de théâtre Black Bazar, Champs Libres 2013, pour souligner l'effet du monologue théâtral.)

\section{Conclusion}

En guise de conclusion, après avoir passé en revue quelques aspects du tragi-comique chez Alain Mabanckou, nous proposons d'inscrire, d’une façon très générale, son écriture dans le giron de la glocalisation.

A l'origine, selon Wikipedia, la glocalisation, traduction du néologisme anglais formé par le mot-valise " globalisation » + " localisation », est l'adaptation spécifique d'un produit ou d'un service à chacun des lieux où il est vendu, ou à chacune des cultures à laquelle il s'adresse (https://en.wikipedia.org/wiki/Glocalization, traduit de l'anglais par PV). Selon Wikipedia encore, il s'agit d'une globalisation auto-limitée, voire régulée grâce à des outils de collaborations sociétaux, qui s'adapte voire s'organise autour des solutions locales. C'est sans doute son premier rôle: agir par la connaissance d'un milieu sur un environnement diversifié. Pour ne donner qu'un exemple concret et banal : tout restaurant chinois dans le monde porte des marques de la glocalisation : à la fois global par l'offre pan-chinoise qu'on trouve partout. Par contre, la localisation se traduit par une adaptation linguistique des Chinois à leur pays d'accueil - il sont capables de maîtriser presque toutes les langues locales au fur et à mesure, mais aussi par le choix du menu, qui est souvent complété par une variété de plats provenant de tel ou tel pays.

Nous pensons que ce concept originaire du monde économique et socioculturel pourrait être utile même dans le domaine de la littérature migrante et de la culture de la migration en général. Que serait alors le produit sus-mentionné dans ce contexte ? A un plus haut niveau, il s'agirait sans doute de l'ensemble des idées autour de la mondialisation et des flux migratoires, qui serait adapté d'abord aux contextes fort différents des pays d'origine des écrivains et de leurs personnages, mais aussi aux grandes métropoles occidentales qui attirent la migration. Un bel exemple de la glocalisation serait le phénomène de la sape. Il s'agit d'une glocalisation curieuse, adaptée d'abord à Brazzaville au Congo, mais apportant l'élément globalisant par le biais des grandes marques de la haute couture telles que Weston ou Gucci. Celles-ci son en même temps adaptées au contexte local : les noirs du Congo recousent leur vêtements sur mesure et les adaptent à leur milieu. Au niveau encore plus élevé de la glocalisation, il est intéressant d’observer comment il est utilisé (voire exploité) par des une compagnies globales comme la Guinness, qui joue également sur la polysémie du mot couleur - noire et locale - dans tous les sens du mot.

Dans le contexte de la littérature, il s'agirait donc d'adapter les grandes questions de la migration et de l'exil, similaires pour tous les écrivains migrants, à leur pays d'accueil. Non seulement pour la raison fort banale et simple que ces auteurs n'auraient souvent pas d'autre expérience, mais aussi pour des stratégies de vente du livre : les lecteurs de la société d'accueil veulent lire ce que les immigrés pensent exactement de leur ville et de leur milieu. Dans le cas de l'humour et du comique, ce sera encore plus remarquable : les lecteur risquent de rire là où il ont une connaissance intime de leur milieu, celui-ci étant employé pour mieux souligner quelques-unes des stratégies du comique. 
La scène de l'arrivée en France d'un Africain est en contraste radical avec celles auxquelles la fiction africaine nous a habitués. Il est déjà passé l'émerveillement devant la complexité du métro d'un Nègre à Paris de Bernard Dadié, il n’y a plus le vertige au milieu de la foule blanche, pas d'éblouissement devant la modernité urbaine européenne comme on peut en voir dans les romans des années 1930 - 1940. Jusque dans ses moindres détails topographiques, Paris est une ville déjà familière au migrant. Avant même d'y avoir physiquement mis les pieds, le migrant est déjà «chez lui » dans l'ancienne métropole coloniale. Le personnage se conduit comme s'il était chez lui - conseillant lui-même au chauffeur (passeur symbolique) le trajet à suivre pour atteindre sa destination finale. Cela témoigne d'un certain changement dans la dynamique de l'échange entre la Métropole et la colonie. Le comique, l'ironie et l'humour en sont souvent des symptômes, mais souvent aussi des moments déclencheurs.

\section{Références bibliographiques}

Cazenave, O. (2004). Afrique sur Seine : une nouvelle génération de romanciers africains à Paris. Paris : L’Harmattan.

Foucault, M. (1966). Les Mots et les choses - Une archéologie des sciences humaines. Paris : Gallimard.

Kundera, M. (1986). Lart du roman. Paris : Gallimard, Collection blanche.

Mabanckou, A. (1998). Bleu Blanc Rouge. Paris : Présence africaine. (2009). Black Bazar. Paris : Seuil.

Moudileno, L. (2006). Parades postcoloniales. La fabrication des identités dans le roman congolais. Paris : Carthala.

Oyono, F. (2006). Le vieux nègre et la médaille. Paris : éd. 10-18.

Pannenborg, W. A. (1955). Écrivains satiriques : caractère et tempérament étudiés d'après des données empruntées à des biographies et à d'autres enquêtes. Paris : PUF.

Sayad, A. (1977). Les trois « âges " de l'émigration algérienne en France. Actes de la recherche en sciences sociales, 15, 59-79.

Simédoh, V. (2012). L'Humour et l'ironie en littérature francophone subsaharienne. Des enjeux critiques à une poétique du rire. New York : Peter Lang.

\section{Webographie}

Champs Libres à... Alain Mabanckou (2013). https://www.youtube.com/watch?v=jg0mbwSIUzk, consulté le 10/10/2016.

Wikipedia Glocalization (2016). https://en.wikipedia.org/wiki/Glocalization, consulté le 11/10/2016. 